\title{
Nonlinear Reduced Order Random Response Analysis of Structures With Shallow Curvature
}

\author{
Adam Przekop* \\ National Institute of Aerospace, Hampton, VA 23666 \\ Stephen A. Rizzi ${ }^{\dagger}$ \\ NASA Langley Research Center, Structural Acoustics Branch, Hampton, VA 23681
}

\begin{abstract}
The goal of this investigation is to further develop nonlinear modal numerical simulation methods for application to geometrically nonlinear response of structures with shallow curvature under random loadings. For reduced order analysis, the modal basis selection must be capable of reflecting the coupling in both the linear and nonlinear stiffness. For the symmetric shallow arch under consideration, four categories of modal basis functions are defined. Those having symmetric transverse displacements (ST modes) can be designated as transverse dominated (ST-T) modes and in-plane dominated (ST-I) modes. Those having anti-symmetric transverse displacements (AT modes) can similarly be designated as transverse dominated (AT-T) modes and in-plane dominated (AT-I) modes. The response of an aluminum arch under a uniformly distributed transverse random loading is investigated. Results from nonlinear modal simulations made using various modal bases are compared with those obtained from a numerical simulation in physical degrees-of-freedom. While inclusion of ST-T modes is important for all response regimes, it is found that the ST-I modes become increasingly important in the nonlinear response regime, and that AT-T and AT-I modes are critical in the autoparametric regime.
\end{abstract}

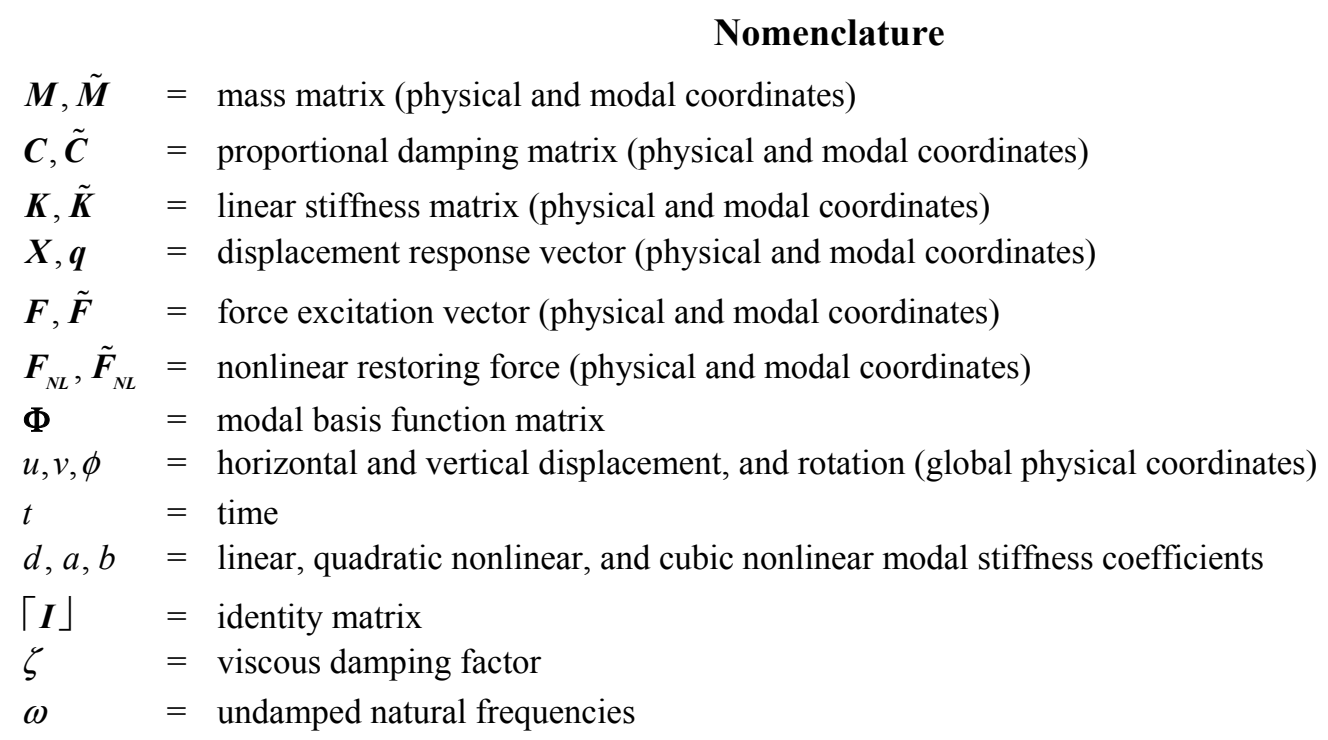

\footnotetext{
${ }^{*}$ Staff Scientist, AIAA Member

${ }^{\dagger}$ Aerospace Engineer, AIAA Associate Fellow
} 


\section{Introduction}

Direct numerical simulation of nonlinear random response in physical degrees-of-freedom (DoFs) is computationally intensive for even the simplest structures. Its use for design of high-cycle-fatigue tolerant aerospace vehicle structures is considered impractical. Accordingly, much effort has been spent in recent years to develop accurate reduced order analyses, such as finite-element-based nonlinear modal numerical simulation, which could be suitable for use in design environments.

Reduced order finite element methods may be viewed as being in one of two categories; those in which the nonlinear modal stiffness is directly evaluated from the nonlinear finite element stiffness matrix (so-called direct methods), and those in which the nonlinear modal stiffness is indirectly evaluated. Direct methods are typically implemented in special purpose finite element codes in which the nonlinear stiffness is known. ${ }^{1-4}$ The only known implementation of a direct method in a commercial code is due to Bathe and Gracewski. ${ }^{5}$ Indirect stiffness evaluation methods are typically implemented for use with commercial finite element codes in which the nonlinear stiffness is unavailable. ${ }^{6,7}$ For both direct and indirect stiffness evaluation approaches, the accuracy of the solution depends on the selection of the modal basis, through which the nonlinear modal stiffness may be determined. The majority of research performed in the field of reduced order numerical simulation of thin-walled aerospace structures has been primarily focused on isotropic planar configurations. ${ }^{1,6-12}$ Through comparison with numerical simulation in physical DoFs, the authors recently demonstrated the ability of a reduced order method to accurately predict geometrically nonlinear random displacement and stress response for a planar isotropic structures, provided that a suitable modal basis is utilized. ${ }^{8}$ However, most practical aerospace applications involve more complex geometries. Thus, the focus of this study is to extend the authors' previous work using an indirect stiffness evaluation approach to determine the effect of modal basis selection on the response of structures with shallow curvature. It is known that such structures may exhibit not only a hardening but also a softening type of nonlinearity ${ }^{13,14}$ which may be non-conservative relative to a linear solution.

For planar configurations, the linear bending and membrane responses are uncoupled. Only the large deflection nonlinear response couples the bending and membrane behavior. Therefore, basis functions derived from a linear eigenanalysis are readily separable into eigenvectors associated with only the bending response and those associated with only the membrane response. Curved structures, on the other hand, exhibit an inherent linear coupling between transverse and in-plane displacements. This coupling is independent of the response magnitude. Similar to the planar configuration, there also exists a coupling of transverse and in-plane displacements resulting from large deflection nonlinearity. Therefore, for reduced order analysis, the modal basis selection must be capable of reflecting the coupling in both the linear and nonlinear stiffness. For the structures under consideration, two categories of modes are defined. Those having symmetric transverse displacements, with anti-symmetric rotations and in-plane displacements, are subsequently referred to as ST modes. Those having anti-symmetric transverse displacements, with symmetric rotations and in-plane displacements, are referred to as AT modes. The ST and AT modes may be further classified according to their dominant behaviors. Mass-normalized eigenvectors dominated by the transverse component will be referred to as a type T mode and those dominated by the in-plane component will be referred to as a type I mode. Consequently, this study is limited to shallow curvatures, allowing the identification of basis functions that are either type T or type I. For the structure considered, type T modes typically have lower natural frequencies than type I modes. Hence, four classes of modes are considered for inclusion in the modal basis, i.e. ST-T, ST-I, AT-T, and AT-I.

The presence of non-linear coupling also implies a possibility of autoparametric resonance, ${ }^{15}$ which is also referred to as autoparametric interaction or internal resonance. ${ }^{16}$ An external loading can directly excite only a motion which is consistent with its characteristics, i.e., symmetric loading acting on a symmetric structure directly excites a symmetric transverse and anti-symmetric in-plane response. However, if sufficiently high nonlinear modal interactions exist, a response behavior not conforming to an external loading may also be initiated. ${ }^{17}$ In other words, an energy flow can occur from a primary system, which is subject to an external excitation, into a secondary system, which is not a subject to an external excitation. Once the secondary system is excited, the energy flow occurs in both directions. ${ }^{18}$ The energy flowing back from the secondary to the primary system in conjunction with a viscous damping can cause the secondary system response to exhibit an intermittent behavior.

An aluminum arch with shallow curvature under transverse loading is considered. Comparisons between nonlinear modal simulations and numerical simulations in physical DoFs are made to determine the proper modal basis selection. Of particular interest is the condition of autoparametric resonance and its effect on the required basis selection. 


\section{Nonlinear numerical simulation in physical DoFs}

The shallow arch with cylindrical curvature was first analyzed with numerical simulation in physical DoFs to give an insight into the characteristic behavior of non-planar structures. In this respect, the simulation is treated as if it were an experimental observation. It will therefore help guide the selection of basis functions used in the reduced order nonlinear modal simulation, and will ultimately be used to validate those results. A clamped-clamped aluminum arch measuring 18 -in. x 1-in. x 0.09 -in (projected length $\mathrm{x}$ width $\mathrm{x}$ thickness) and 81.25 -in. radius of curvature was considered, as shown in Figure 1. This geometry gave a radius/span ratio of approximately 4.5. The following material properties were used:

$$
E=10.6 \times 10^{6} \mathrm{psi}, \quad G=4.0 \times 10^{6} \mathrm{psi}, \quad \rho=2.588 \times 10^{-4} \frac{\mathrm{lb_{f }}-\mathrm{s}^{2}}{i \mathrm{in}^{4}} .
$$

Mass proportional damping was specified corresponding to critical damping of $2.0 \%$ for the first symmetric transverse mode (at $258 \mathrm{~Hz}$ ). A random load generation process previously presented ${ }^{9}$ was applied, giving a flat excitation spectrum from 0 to $1500 \mathrm{~Hz}$. The arch was uniformly loaded along its span in the vertical direction, irrespective of the deformation, i.e. follower forces were not utilized.

The finite element program ABAQUS (version 6.5-1) was used to generate nonlinear displacement time histories. The double precision explicit integration scheme with an adaptive time integration step (referred to as "element by element" in ABAQUS) was utilized for all analyses. A total time of $2.1384 \mathrm{~s}$ was simulated for each loading level considered, and the first $0.5 s$ was removed to eliminate the transient response. The ABAQUS model consisted of 144 B21 beam elements. All displacement results are presented in the global coordinate system as horizontal $(u)$ and vertical $(v)$ components.

The responses to four different excitation levels yielding slightly to highly nonlinear response characteristics were computed. The $u$ and $v$ displacement power spectral densities (PSDs) were examined at two locations, namely at the projected quarter-span of the arch (4.5 in. from the clamped boundary), presented in Figure 2 and Figure 3, and at the mid-span (9.0 in. from the clamped boundary), presented in Figure 4 and Figure 5. Displacement responses at the quarter-span location (Figure 2 and Figure 3) clearly indicate a significant change in the PSD characteristics when passing from the $0.4608 \mathrm{lb} /$ in. to the $0.6517 \mathrm{lb} /$ in. excitation level. In particular, the emergence of a peak at approximately $158 \mathrm{~Hz}$ is noted. At the mid-span location, the $v$ displacement PSD (Figure 4) does not indicate this change in character. Examination of mid-span $u$ displacement PSD (Figure 5), however, indicates negligible response amplitude at the $0.2304 \mathrm{lb} /$ in. excitation level, followed by a significant jump in amplitude at and above the $0.6517 \mathrm{lb} / \mathrm{in}$. excitation level. The $0.4608 \mathrm{lb} / \mathrm{in}$. excitation level appears to produce a response in a transition region.

The jump in response in Figure 5 was investigated in further detail. Figure 6 presents mid-span $u$ displacement time histories ranging from the $0.2304 \mathrm{lb} / \mathrm{in}$. to $1.3033 \mathrm{lb} / \mathrm{in}$. excitation levels. At the excitation level of 0.2304 $\mathrm{lb} / \mathrm{in}$., the mid-span $u$ response is negligible. Previous work indicated this to be the case for a planar isotropic and uniformly loaded beam, regardless the excitation level applied. ${ }^{8}$ For the loading level of $0.6517 \mathrm{lb} / \mathrm{in}$., the mid-span $u$ displacement of the arch exhibits intermittent damped transients. At the excitation level of $1.3033 \mathrm{lb} / \mathrm{in}$., the motion is of a permanent nature. A few instantaneous snapshots of a full-field displacement along the span of the arch for the $0.6517 \mathrm{lb} / \mathrm{in}$. loading were studied to determine the nature of this motion. Snapshots were chosen from the evolved $u$ displacement time history shown in Figure 7 such that, the center point displacement was negative (left of the neutral position, $\mathrm{t}=1.701 \mathrm{~ms}$ ), positive (right of the neutral position, $\mathrm{t}=4.148 \mathrm{~ms}$ ) and nearly zero (at the neutral position, $\mathrm{t}=3.225 \mathrm{~ms}$ ). Snapshots of the $v$ and $u$ displacements along the arch span for these three conditions are presented in Figure 8 and Figure 9, respectively. When the mid-span $u$ response is zero $(\mathrm{t}=3.225 \mathrm{~ms})$, the $v$ displacement is symmetric and $u$ displacement is anti-symmetric. It is seen in both figures that symmetry of the $v$ displacement response and anti-symmetry of the $u$ displacement response is only preserved when the mid-span $u$ displacement is zero. When the mid-span $u$ displacement response is positive $(\mathrm{t}=1.701 \mathrm{~ms})$, the $v$ displacement response is no longer symmetric and its maximum occurs at approximately the quarter-span point from the right end. When the mid-span $u$ displacement response is negative $(\mathrm{t}=4.148 \mathrm{~ms})$, the $v$ displacement response is also no longer symmetric, with its maximum at approximately the quarter-span point from the left end. These observations lead to the conclusion that both $u$ and $v$ displacements are a superposition of both symmetric and anti-symmetric behaviors.

Due to the symmetry of the arch geometry and the loading distribution, it can also be concluded that there exists no direct form of excitation that would initiate the anti-symmetric $v$ and/or symmetric $u$ displacement responses, i.e., non-symmetric $v$ displacement and/or any mid-span $u$ motion. Therefore this behavior must be initiated by energy transfer from the primary system, which is consistent with the geometry and loading. Recalling Section I, such an observation fulfills a definition of an autoparametric resonance. The analysis in physical DoFs, however, is unable to provide detailed insight as to what modes contribute to the anti-symmetric behavior. Certainly, inspection of 
Figure 2 and Figure 3 can provide some clues regarding the new frequency components introduced with the antisymmetric $v$ displacement response (i.e. at approximately $160,520,1070 \mathrm{~Hz}$ ). However, since the response is nonlinear, characterization of this nonlinear behavior in terms of linear modes is appropriate only in the context of a nonlinear modal simulation.

Founded on experience built upon flat and isotropic thin-walled structures analysis, there exists a perception that a nonlinear analysis is generally an attempt to mitigate conservativeness or over-design that would result from a linear analysis. However, nonlinear curved and/or anisotropic structural analysis can result in a solution that will not be upper-bounded by a linear analysis. ${ }^{13,14}$ Figure 10 and Figure 11 present linear and nonlinear vertical root mean square (RMS) displacement results at the mid-span and quarter-span locations, respectively. It is clearly seen that for the shallow arch analyzed herein, the displacements at both selected locations obtained from the nonlinear analysis will exceed the results obtained form the linear calculations. Hence, this structure exhibits a spring softening nonlinearity and is non-conservative relative to the linear system.

\section{Reduced order numerical simulation}

The nonlinear reduced order numerical simulation analysis consists of several parts. ${ }^{8}$ The linear eigenvectors, commonly employed as basis functions, are first obtained from a commercial finite-element program (in this study ABAQUS v.6.5-1). Following a transformation of the nonlinear system of equations to modal coordinates, the modal stiffness coefficients are evaluated and the resulting coupled system of equations is numerically integrated to obtain the modal displacement time history. The modal solution is transformed back to physical coordinates and can be post-processed as desired. It should be pointed out that for the purpose of analyzing non-planar structures, the formulation previously presented ${ }^{7,8,11}$ has not been redeveloped. However, the guidelines for the selection of basis functions must be substantially revised.

\section{A. Modal Coordinate Transformation}

The equations of motion of the nonlinear system in physical DoFs may be written as

$$
\boldsymbol{M} \ddot{\boldsymbol{X}}(t)+\boldsymbol{C} \dot{\boldsymbol{X}}(t)+\boldsymbol{F}_{N L}(\boldsymbol{X}(t))=\boldsymbol{F}(t) .
$$

where $\boldsymbol{M}$ and $\boldsymbol{C}$ are the mass and proportional damping matrices, respectively, and $\boldsymbol{X}$ and $\boldsymbol{F}$ are the displacement response and force excitation vectors, respectively. The nonlinear restoring force $\boldsymbol{F}_{N L}$ contains the linear, quadratic and cubic stiffness terms.

A set of coupled modal equations with reduced degrees-of-freedom is first obtained by applying the modal coordinate transformation $\boldsymbol{X}=\boldsymbol{\Phi} \boldsymbol{q}$ to Equation (1), where $\boldsymbol{q}$ is the modal displacement response vector. The modal basis function matrix $\Phi$ is typically formed from the eigenvectors obtained from Equation (1) using only the linear stiffness. Generally, a small set of $L$ basis functions are included giving

$$
\tilde{\boldsymbol{M}} \ddot{\boldsymbol{q}}(t)+\tilde{\boldsymbol{C}} \dot{\boldsymbol{q}}(t)+\tilde{\boldsymbol{F}}_{N L}\left(q_{1}(t), q_{2}(t), \ldots, q_{L}(t)\right)=\tilde{\boldsymbol{F}}(t)
$$

where the tilde superscript represents modal quantities, and

$$
\tilde{\boldsymbol{M}}=\boldsymbol{\Phi}^{T} \boldsymbol{M} \boldsymbol{\Phi}=\lceil\boldsymbol{I}\rfloor \quad \tilde{\boldsymbol{C}}=\boldsymbol{\Phi}^{T} \boldsymbol{C} \boldsymbol{\Phi}=\left\lceil 2 \zeta_{r} \omega_{r}\right\rfloor \quad \tilde{\boldsymbol{F}}_{N L}=\boldsymbol{\Phi}^{T} \boldsymbol{F}_{N L} \quad \tilde{\boldsymbol{F}}=\boldsymbol{\Phi}^{T} \boldsymbol{F} .
$$

\section{B. Indirect Stiffness Evaluation Method}

The previously developed indirect stiffness evaluation method is applied. ${ }^{7,8,11}$ To summarize, the nonlinear force vector in Equation (2) may be written in the form

$$
\tilde{F}_{N L}\left(q_{1}, q_{2}, \ldots, q_{L}\right)=\sum_{j=1}^{L} d_{j}^{r} q_{j}+\sum_{j=1}^{L} \sum_{k=j}^{L} a_{j k}^{r} q_{j} q_{k}+\sum_{j=1}^{L} \sum_{k=j}^{L} \sum_{l=k}^{L} b_{j k l}^{r} q_{j} q_{k} q_{l} \quad r=1,2, \ldots, L
$$

where $d, a$, and $b$ are the linear, quadratic nonlinear, and cubic nonlinear modal stiffness coefficients, respectively. This form reduces the problem of determining the nonlinear stiffness from one in which a large set of simultaneous nonlinear equations must be solved to one involving simple algebraic relations. The algebraic relations are obtained 
by solving a series of nonlinear static problems with prescribed displacement fields. ${ }^{7,8,11}$ Note that the indirect procedure, contrary to the direct methods, ${ }^{1-3}$ does not neglect in-plane inertia of the system.

\section{Eigenanalysis}

As mentioned briefly in Section III.A, a reduced order method usually employs a subset of selected eigenvectors as a set of basis functions to be used in a reduced order stiffness evaluation procedure. As expected and explained in Section I, the identification and classification of these eigenvectors for a shallow structure turns out to be more involved than for planar configurations. It is helpful to categorize the linear eigenvectors that constitute the modal basis by their symmetry. For the shallow shell under consideration two categories are defined. Those having symmetric transverse displacements, with symmetric rotations and in-plane displacements, are subsequently referred to as ST modes. Those having anti-symmetric transverse displacement with symmetric rotations and in-plane displacements, are referred to as AT modes. Each eigenvector consists of both transverse and in-plane components, therefore an additional criteria is needed in an attempt to recognize whether a particular mode will be contributing more to a transverse or in-plane response component. In this work the following classification was adopted: For each mass normalized eigenvector, the maximum transverse and in-plane components were determined. If the maximum transverse component was greater than the maximum in-plane component, the mode was classified as type $\mathrm{T}$ - transverse dominated. If the maximum in-plane component was greater than the maximum transverse component, the mode was classified as type I - in-plane dominated. Hence, four classes of modes are considered for inclusion in the modal basis, i.e., ST-T, ST-I, AT-T, and AT-I. Note that this scheme for classifying mode type is applicable only to the shallow curvatures under consideration here. For deeper curvatures, an alternative classification scheme would likely be necessary. It should be noted that either the full span model, or a semi-span model analyzed with both symmetric and anti-symmetric mid-span boundary conditions, are needed to obtain the above suite of eigenvectors.

The identical ABAQUS model was used for both the physical DoFs solution and eigenanalysis for the reduced order numerical simulation. Selected modes with their ordering numbers, corresponding natural frequencies, and the above-introduced classification are shown in Table 1. For the structure considered, type T modes typically have lower natural frequencies than type I modes. Vertical displacement, rotation, and horizontal displacement components of the lowest natural frequency eigenvectors of each of the four categories defined above are shown in Figure 12 to Figure 15.

At this point it is also worthwhile to note that the concept of so-called companion ${ }^{10}$ or dual ${ }^{11,12}$ modes developed for a planar structures is no longer needed nor relevant. These bases were previously introduced to obtain membrane behavior corresponding to bending modes of the flat structure. Since the modes are linearly coupled for curved structures, these degrees of freedom are automatically reflected in the basis.

\section{Modal Stiffness Relationships}

It was previously shown ${ }^{8}$ that the linear modal stiffness obtained from the indirect stiffness evaluation method is equivalent to that obtained via a standard modal analysis, that is,

$$
\boldsymbol{d}=\tilde{\boldsymbol{K}}=\boldsymbol{\Phi}^{T} \boldsymbol{K} \boldsymbol{\Phi}=\left\lceil\omega_{r}^{2}\right\rfloor
$$

As expected, there is zero modal coupling through the linear modal stiffness. Additionally, certain relationships exist between significant quadratic and cubic nonlinear stiffness terms. ${ }^{7,8}$ For the quadratic terms, these are

$$
\begin{aligned}
a_{12}^{1} & =2 a_{11}^{2} \\
2 a_{22}^{1} & =a_{12}^{2}
\end{aligned}
$$

For the cubic terms, these are

$$
\begin{aligned}
b_{122}^{1} & =b_{112}^{2} \\
3 b_{222}^{1} & =b_{122}^{2} \\
3 b_{111}^{2} & =b_{112}^{1}
\end{aligned}
$$

Without citing particular values, these relationships are maintained for the present problem.

It is interesting to note that the quadratic stiffness terms for like indices, i.e. the $a_{j j}$ terms, are essentially zero for

both AT-T and AT-I modes, and are generally non-zero for both ST-T and ST-I modes. Thus, only diagonal terms 
corresponding to ST modes contribute to spring softening. Cross quadratic stiffness coefficients, i.e. the $a_{j k}$ terms, may however also contribute to spring softening. Table 2 lists the cross quadratic coefficients for the first ST-T, STI, AT-T, and AT-I modes. A strong coupling is noted between the ST-T and ST-I mode pair, the ST-I and AT-I mode pair, and the AT-T and ST-I mode pair. A weak coupling is noted between the ST-T and AT-T mode pair, and the ST-T and AT-I mode pair. The ST-T and AT mode pairs are not coupled through the quadratic stiffness. The cubic cross stiffness coefficients for these mode combinations are also indicated in Table 2. It is noted that only the ST-T and ST-I mode pair, and AT-T and AT-I mode pair indicate significant values for both the $b_{j j k}$ and

$b_{j k k}$ terms. The other pairs indicate significant values for only one of the terms. The significance is that there is a higher degree of coupling of cubic stiffness terms for the ST-T and ST-I mode pair, and AT-T and AT-I mode pair, than for the other combinations. Hence, even if the higher frequency ST-I and AT-I modes fall outside of the excitation frequency bandwidth, they may have a significant effect on the solution as they are strongly coupled to the ST-T and AT-T modes, respectively.

\section{E. Reduced order analysis results}

For the shallow curvature considered, two factors affected the initial modal basis selection. The first was earlier experience with symmetric planar structures under symmetric transverse loadings where the initial basis consisted of low frequency bending modes. ${ }^{7-12}$ For the curved configuration, an analogous basis consisting of transverse component dominated (type T) modes was chosen. Like the earlier experience, the addition of higher frequency inplane dominated modes (type I) was expected to improve the prediction of peak magnitudes and their broadening in a highly nonlinear regime. ${ }^{8}$ The second selection factor was based on observations made through the simulation in physical DoFs. There, the need for both ST-T and AT-T modes was indicated for autoparametric resonance conditions. Thus the modal bases chosen consisted of all four types of modes. In the following, an abbreviated convention describing the set of modes applied in a particular analysis was used. The convention consists of a fourdigit sequence containing the number of included ST-T, ST-I, AT-T, and AT-I modes, in that order. For example, 4044 indicates a basis consisting of four ST-T, zero ST-I, four AT-T, and four AT-I modes.

Two excitation levels were selected for investigation; $0.2304 \mathrm{lb} / \mathrm{in}$. was shown to give rise to a slightly nonlinear response, and $0.6517 \mathrm{lb} /$ in. produced a highly nonlinear response. As illustrated in Figure 6, the lower excitation level does not produce an autoparametric response, while the higher level produces an intermittent autoparametric behavior. For both loading levels, the following suite of basis functions was considered. The 4040 basis was selected as the baseline as it contained only type T modes, i.e. four ST-T and four AT-T modes. The 4440 basis was used to determine the effect of ST-I modes on the baseline. Similarly, the 4044 basis was used to determine the effect of AT-I modes on the baseline. The effect of both types of in-plane dominated modes on the baseline was studied using the 4444 basis. Two additional bases were considered to investigate the contribution of only ST-T modes in the 4000 basis, and the contribution of only AT-T modes, in the 0040 basis. These last two bases were ideal for investigating the response to direct excitation. The ability to interrogate the nonlinear response in this manner is a unique capability of the nonlinear modal simulation. An analysis in physical DoFs can only approximate this ability by band-limiting the excitation field. The effect of nonlinear modal coupling however remains in that case.

Having the nonlinear force vector in Equation (4) fully defined with any set of the basis functions described above, the coupled modal nonlinear equations of motion given by Equation (2) were numerically integrated using a fourth-order Runge-Kutta method. ${ }^{19}$ Consistent with the analysis in physical DoFs, a total time of $2.1384 s$ was simulated and the initial $0.5 s$ of transient response was removed. A fixed time integration step of $2 \mu s$ was used.

The resulting modal displacement time histories were transformed back to physical coordinates using the inverse modal transformation.

Horizontal and vertical displacement PSDs obtained via reduced order analysis were compared with those obtained via the analysis in physical DoFs at two locations along the span of the arch, namely at the center and quarter-span nodes. The need to select more than one validation point was based on the observation from the analysis in physical DoFs that the change in nonlinear response manifests itself in different ways at different locations. At the quarter span location, the emergence of an additional peak was noted in Figure 2 and Figure 3. At the mid-span node, the $u$ displacement component exhibited a sudden jump in response level with the onset of autoparametric resonance.

Figure 16 to Figure 18 present the comparison of the reduced order solutions obtained with different bases and the solution in physical DoFs at the $0.2304 \mathrm{lb} /$ in. excitation level. For these and subsequent plots, significant peaks are annotated with their corresponding modal component to aid the discussion. It should be understood that these 
annotations do not imply the presence of linear modes in the nonlinear response. The $u$ displacement at the midspan is not shown, since all analyses yielded numerical zero. All reduced order displacement PSDs compare well with the physical DoFs solution and demonstrate behaviors due solely to ST modes. This is evidenced by the fact that the inclusion of AT-T and AT-I modes in the basis do not alter the computed response, e.g. compare 4000 with 4040 and 4044 . In fact, the 0040 basis resulted in a numerically zero response at both locations for both response components, indicating that the AT modes are not directly excited by the externally applied loading. The only noticeable difference between modal bases is apparent at the quarter-span location in the first sub-harmonic of the lowest ST-T mode $(133 \mathrm{~Hz})$. Upon close inspection, it is seen that the behavior is represented slightly better with the $44 \mathrm{xx}$ bases (4444 and 4440), than it is with either of the 40xx bases (4044, 4040, and 4000). Therefore, even for this slightly nonlinear response regime, the inclusion of ST-I modes is shown to improve the behavior related to the ST-T modal response. As discussed in Section III.D, this is due to the cross coupling of cubic stiffness terms between the ST-T and ST-I mode pairs. Note that if the excitation bandwidth were sufficiently increased, the ST-I modes would also be directly excited. From this load level, we conclude that the ST-T modes (though direct excitation) and ST-I modes (through coupling with ST-T modes) constitute the primary system.

Figure 19 to Figure 26 show the results obtained at an excitation level of $0.6517 \mathrm{lb} / \mathrm{in}$. For clarity, each set of solutions is displayed in two separate figures. One figure indicates the physical DoFs, 4444, and 4044 solutions. The second figure shows the physical DoFs, 4440, 4040, and 4000 solutions.

At the mid-span location, the $v$ displacement response (Figure 19 and Figure 20) is dominated by the peak originating from the first ST-T mode. The response is best modeled by the 4444 and 4440 bases. Since the PSD response does not indicate a presence of peaks originating from anything other than ST-T modes, this result is found consistent with the conclusions based on $0.2304 \mathrm{lb} / \mathrm{in}$. case, where the comparison of $44 \mathrm{xx}$ bases with the physical DoFs solution was found superior to the 40xx bases. The 40xx bases $(4044,4040$, and 4000) over-predict the degree of nonlinearity. This is evidenced through excessive stiffening (peaks moved to the higher frequencies compared to physical DoFs) of the first, third and fourth ST-T-related peaks, and excessive broadening, especially of the fourth peak. The second ST-T-related behavior is not significantly present at this location. The solution for the 0040 basis was numerically zero, indicating that the AT-T modes were not directly excited.

Mid-span $u$ displacement results (Figure 21 and Figure 22) offer a different perspective. The response exhibits peaks originating from the AT-T modes. The 0040 basis yielded a numerically zero response, indicating that the AT-T modes are not directly excited by the externally applied loading. The excitation provided to the AT-T modes is therefore applied indirectly through autoparametric resonance. Thus, the AT-T modes (through autoparametric resonance) and the AT-I modes (through coupling with the AT-T modes) constitute the secondary system. The modal bases containing ST and both AT-T and AT-I modes are therefore expected to correspond best with the physical DoFs solution for this condition. The solution for basis 4044, shown in Figure 21, corresponds fairly well with the physical DoFs solution. However, the magnitude of the first AT-T peak is too low, and it demonstrates over-stiffening. Further, the comparison beyond approximately $750 \mathrm{~Hz}$ frequency range is deficient. The addition of ST-I modes in basis 4444 activates the ST-T to ST-I coupling and improves the comparison throughout the frequency range. Bases which do not include AT-I modes (4440 and 4040) suffer as the AT-T to AT-I coupling is not represented, see Figure 22. The 4440 basis captures the behavior of the first AT-T peak better than the 4040 basis, likely due to the ST-T to ST-I coupling. Beyond about $300 \mathrm{~Hz}$, both 4440 and 4040 bases compare poorly, as the response level generally under-predicts the physical DoFs solution except in the frequency range between 1050 and $1200 \mathrm{~Hz}$, where the contribution of the third AT-T is exaggerated. It can be concluded that the higher frequency modes of both kinds, ST-I and AT-I, are needed for an accurate mid-span horizontal displacement prediction when the structure undergoes autoparametric resonance. However, inclusion of the AT-I modes is more important than inclusion of the ST-I modes, as evidenced by the improved comparison of the 4044 basis relative to the comparison of the 4440 basis. This observation further substantiates the importance of the coupling between the ST-T and ST-I modes, and between the AT-T and AT-I modes. Note that basis 4000 resulted in an essentially zero response, indicating that the basis is incapable of representing the secondary system and capturing the autoparametric interactions.

At the quarter-span location, the $v$ displacement component (Figure 23 and Figure 24) is best captured by 4444 basis. Basis 4000 does not have means of representing the anti-symmetric behavior, and consequently provides a solution that fails to capture the behavior of the secondary system. This is particularly apparent in the lack of the peak originating from the first AT-T mode, including its sub-harmonic peak. Among those bases that are capable of reflecting the anti-symmetric behavior, basis 4040 is of the lowest quality. It poorly represents the peak originating from the first AT-T mode in that it is over-stiff (its peak magnitude is too small and is shifted toward higher frequency). Additionally, its AT-T sub-harmonic is too small while the contribution of the third AT-T mode is exaggerated. The quality of solutions from bases 4440 and 4044 consistently falls between the best (4444) and the 
worst (4040). Overall, basis 4440 matches the peak magnitudes and broadening better than the 4044 basis. The exception is in the frequency range related to the third AT-T mode (approximately $1050-1150 \mathrm{~Hz}$ ), in which the solution from the 4440 basis is vastly exaggerated, while the solution from the 4044 basis over-predicts this peak only slightly.

At the quarter-span, the $u$ displacement component (Figure 25 and Figure 26) obtained with 4444 basis is again superior to all the others. It very accurately matches peaks originating from both the ST-T and AT-T modes. It is particularly worthwhile to note that 4444 basis is the only one that does not exhibit over-stiffening of the first AT-T related peak, and accurately captures the second and the third AT-T related peaks. Remarks made regarding the performance of 4440, 4040, and 4000 solutions for the vertical displacement component apply to the horizontal displacement component as well. The only exception is that the 4044 basis under-predicts the peak originating from the third AT-T mode for the $u$ displacement component.

\section{Conclusions}

The effect of modal basis selection on the displacement response predicted using a nonlinear modal simulation was investigated. For the shallow arch considered, it was found that:

$>$ At a sufficiently high excitation level autoparametric resonance can exist;

$>$ For a symmetric curved structure and symmetric loading distribution, the primary system consists of ST-T and ST-I modes and these modes may be directly excited by external loadings with sufficient bandwidth;

$>$ For a symmetric curved structure and symmetric loading, modes AT-T and AT-I are never directly excited (regardless of the excitation level and bandwidth); these modes constitute the secondary system and are only indirectly excited through the nonlinear modal interactions at a sufficiently high response regime;

> For non-planar symmetric structures which may exhibit autoparametric resonance, the inclusion of both ST$\mathrm{T}$ and AT-T modes is the necessary minimum to represent directly and indirectly loaded components;

$>$ Higher frequency mode variants (ST-I and AT-I) provide a significant enhancement of the solution quality in the highly nonlinear/autoparametric regime; for all the cases studied at the high excitation level, inclusion of all mode variants (ST-T, ST-I, AT-T, and AT-I) always provided the solution that compared best with the physical DoFs analysis;

$>$ The inclusion of all mode variants at any location or excitation level does not adversely affect the response prediction; when mode identification is difficult, it should be always "safe" to expand the set of basis functions;

$>$ Modal pairs: ST-T with ST-I, and AT-T with AT-I exhibit the strongest cubic coupling; therefore in the highly nonlinear/autoparametric regime, modes ST-I appear to enhance ST-T-originating response features, while AT-I appear to enhance AT-T-originating response features;

$>$ Validation of a reduced order solution versus physical DoFs analysis and/or experimental results should be performed at more than one location on the structure and/or by inspection of more than one response component; e.g., if only transverse deflection at the center point would be considered, basis 4000 might have appeared sufficient;

$>$ Nonlinear analysis of shallow curvature structures may yield non-conservative results when compared with linear analysis.

$>$ The ability to interrogate the nonlinear response by altering the modal basis is a unique capability of the nonlinear modal simulation; analyses in physical DoFs will always reflect nonlinear modal coupling.

The results presented in this paper were for a particular shallow curvature. A possible area for future work is to determine the onset of autoparametric resonance as a function of curvature. A related interest is to extend the assessment of modal basis selection to structures having greater curvature and to those having linear coupling due to material anisotropy. At a minimum, it is expected that the present modal classification scheme would need modification for these cases. 


\section{References}

${ }^{1}$ Mei, C., Dhainaut, J.M., Duan, B., Spottswood, S.M., and Wolfe, H.F., "Nonlinear random response of composite panels in an elevated thermal environment," Air Force Research Laboratory AFRL-VA-WP-TR-2000-3049, Wright-Patterson Air Force Base, OH, October 2000.

${ }^{2}$ Przekop, A., Guo, X., Azzouz, M.S., and Mei, C., "Reinvestigation of nonlinear random response of shallow shells using finite element modal formulation," Proceedings of the 45th AIAA/ASME/ASCE/AHS/ASC Structures, Structural Dynamics and Materials Conference, AIAA-2004-1553, Palm Springs, CA, 2004.

${ }^{3}$ Przekop, A., "Nonlinear response and fatigue estimation of aerospace curved surface panels to combined acoustic and thermal loads," Ph.D. Dissertation, Old Dominion University, Norfolk, VA, 2003.

${ }^{4}$ Ribeiro, P., "Modal Interactions in Shallow Arches," Proceedings of the 7th International Conference on Computational Structures Technology, Lisbon, Portugal, 2004, B.H.V. Topping, et al. (ed.), Civil-Comp Press, Stirling, Scotland.

${ }^{5}$ Bathe, K.J. and Gracewski, S., "On Nonlinear Dynamic Analysis using Substructuring and Mode Superposition," Computers and Structures, Vol. 13, No. 1981, pp. 699-707.

${ }^{6}$ McEwan, M.I., Wright, J.R., Cooper, J.E., and Leung, Y.T., "A finite element/modal technique for nonlinear plate and stiffened panel response prediction," Proceedings of the 42nd AIAA/ASME/ASCE/AHS/ASC Structures, Structural Dynamics, and Materials Conference, AIAA-2001-1595, Seattle, WA, 2001.

${ }^{7}$ Muravyov, A.A. and Rizzi, S.A., "Determination of nonlinear stiffness with application to random vibration of geometrically nonlinear structures," Computers and Structures, Vol. 81, No. 15, 2003, pp. 1513-1523.

${ }^{8}$ Rizzi, S.A. and Przekop, A., "The effect of basis selection on thermal-acoustic random response prediction using nonlinear modal simulation," Proceedings of the 45th AIAA/ASME/ASCE/AHS/ASC Structures, Structural Dynamics and Materials Conference, AIAA-2004-1554, Palm Springs, CA, 2004.

${ }^{9}$ Rizzi, S.A. and Muravyov, A.A., "Comparison of nonlinear random response using equivalent linearization and numerical simulation," Structural Dynamics: Recent Advances, Proceedings of the 7th International Conference, Vol. 2, Southampton, UK, 2000, N.S. Ferguson, et al. (ed.), pp. 833-846.

${ }^{10}$ Hollkamp, J.J., Gordon, R.W., and Spottswood, S.M., "Nonlinear sonic fatigue response prediction from finite element modal models: a comparison with experiments," Proceedings of the 44th AIAA/ASME/ASCE/AHS/ASC Structures, Structural Dynamics, and Materials Conference, AIAA-2003-1709, Norfolk, VA, 2003.

${ }^{11}$ Mignolet, M.P., Radu, A.G., and Gao, X., "Validation of reduced order modeling for the prediction of the response and fatigue life of panels subjected to thermo-acoustic effects," Structural Dynamics: Recent Advances, Proceedings of the 8th International Conference, Southampton, UK, 2003, M.J. Brennan, et al. (ed.).

${ }^{12}$ Radu, A.G., Yang, B., Kim, K., and Mignolet, M.P., "Prediction of the dynamic response and fatigue life of panels subjected to thermo-acoustic loading," Proceedings of the 45th AIAA/ASME/ASCE/AHS/ASC Structures, Structural Dynamics and Materials Conference, AIAA-2004-1557, Palm Springs, CA, 2004.

${ }^{13}$ Kobayashi, Y. and Leissa, A.W., "Large amplitude free vibration of thick shallow shells supported by shear diaphragms," Journal of Non-linear Mechanics, Vol. 30, No. 1, 1995, pp. 57-66.

${ }^{14}$ Przekop, A., Azzouz, M.S., Guo, X., Mei, C., and Azrar, L., "Finite Element Multiple-Mode Approach to Nonlinear Free Vibrations of Shallow Shells," AIAA Journal, Vol. 42, No. 11, 2004, pp. 2373-2381.

${ }^{15}$ Tondl, A., Ruijgrok, T., Verhulst, F., and Nabergoj, R., Autoparametric resonance in mechanical systems. Cambridge University Press, Cambridge, UK, 2000.

${ }^{16}$ Nayfeh, A.H. and Mook, D.T., Nonlinear Oscillations. Wiley, New York, 1995.

${ }^{17}$ Ibrahim, R.A., Parametric random vibration. Wiley, New York, 1985.

${ }^{18}$ Matthew, C., Introduction to linear, parametric, and nonlinear vibrations. Chapman and Hall, New York, 1990.

${ }^{19}$ Press, W.H., Teukolsky, S.A., Vetterling, W.T., and Flannery, B.P., "Numerical recipes, the art of scientific computing," CDROM v2.10, Cambridge University Press, 2002. 


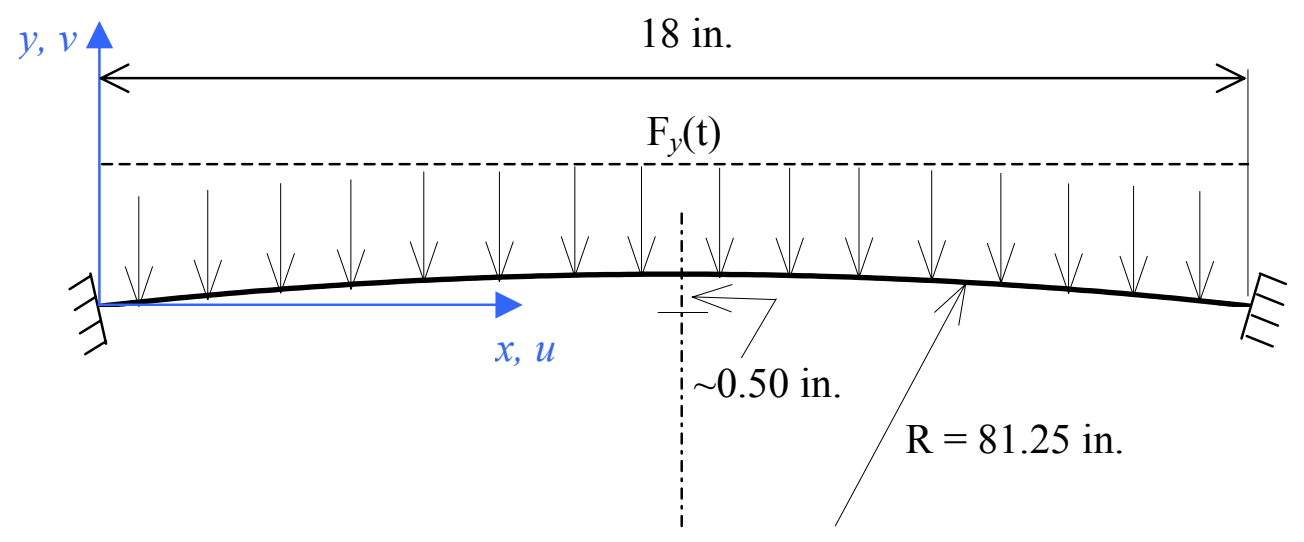

Figure 1: Geometry of shallow arch.

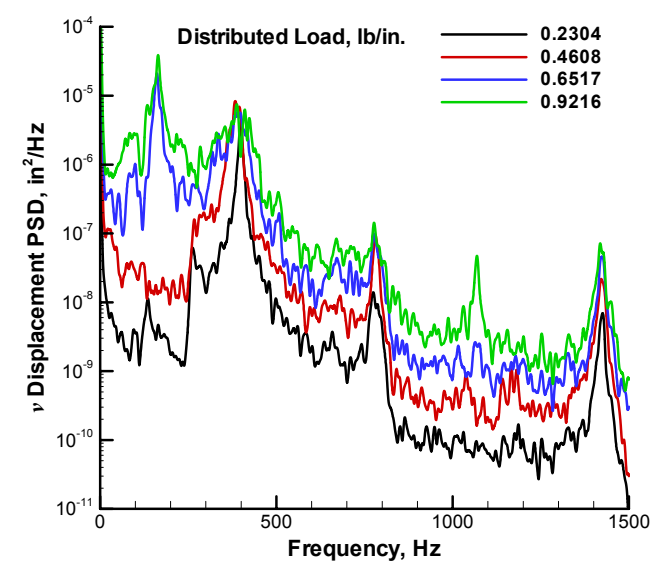

Figure 2: Vertical displacement PSD at quarterspan location.

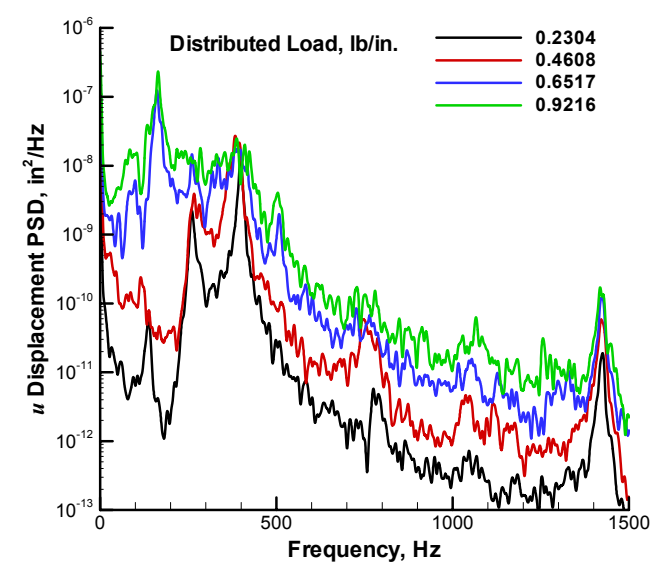

Figure 3: Horizontal displacement PSD at quarter-span location.

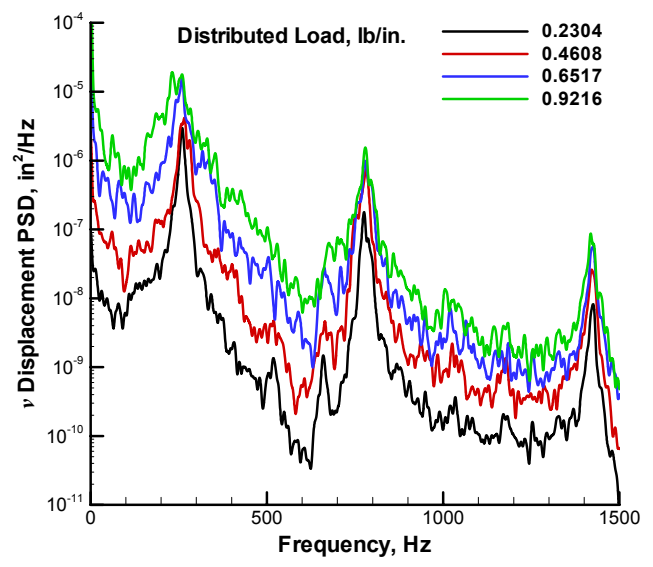

Figure 4: Vertical displacement PSD at mid-span location.

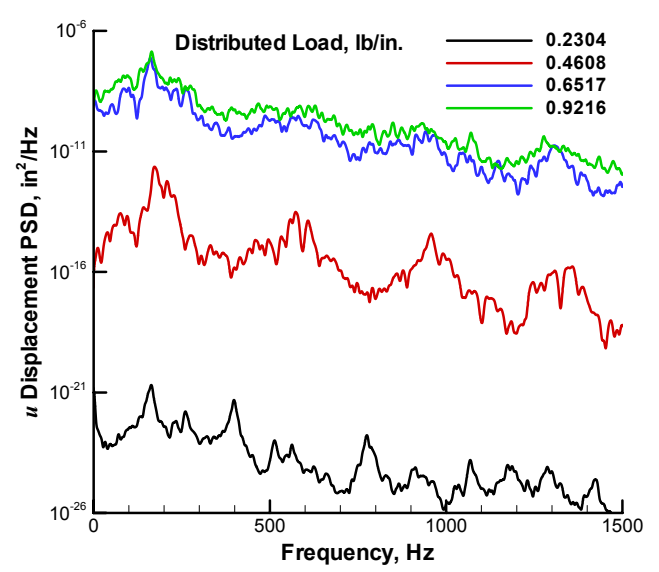

Figure 5: Horizontal displacement PSD at midspan location. 


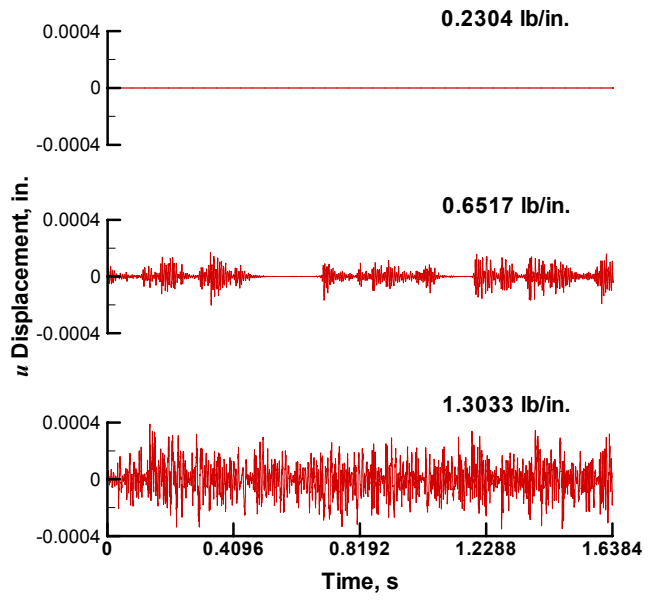

Figure 6: Mid-span horizontal displacement response time histories.

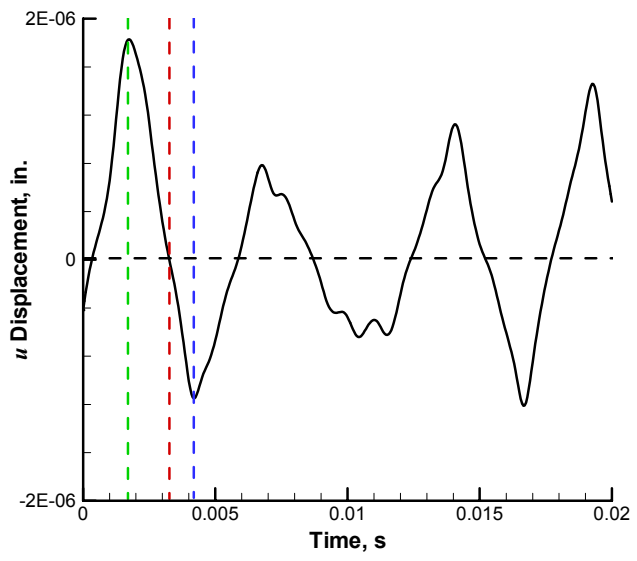

Figure 7: Mid-span horizontal displacement time history at $0.6517 \mathrm{lb} / \mathrm{in}$. distrib. load.

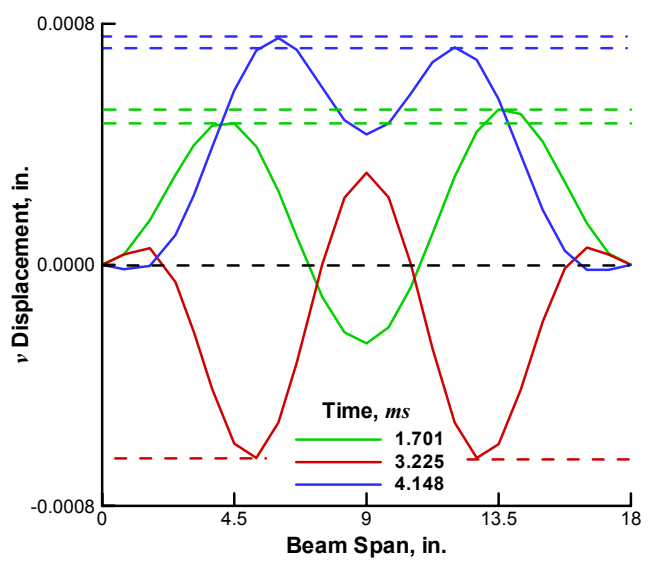

Figure 8: Vertical displacement field for selected time steps at $0.6517 \mathrm{lb} / \mathrm{in}$. distrib. load.

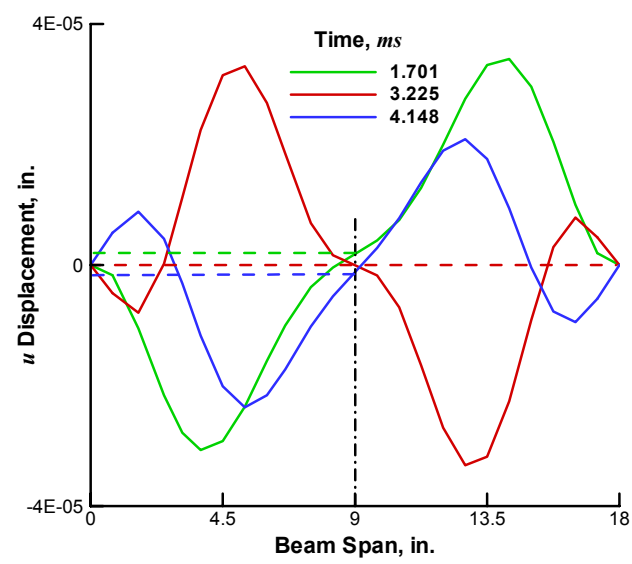

Figure 9: Horizontal displacement field for selected time steps at $0.6517 \mathrm{lb} /$ in. distrib. load.

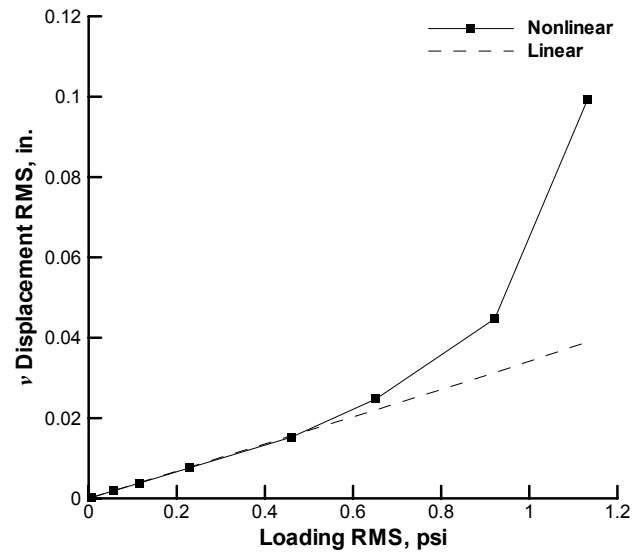

Figure 10: Mid-span $v$ displacement RMS.

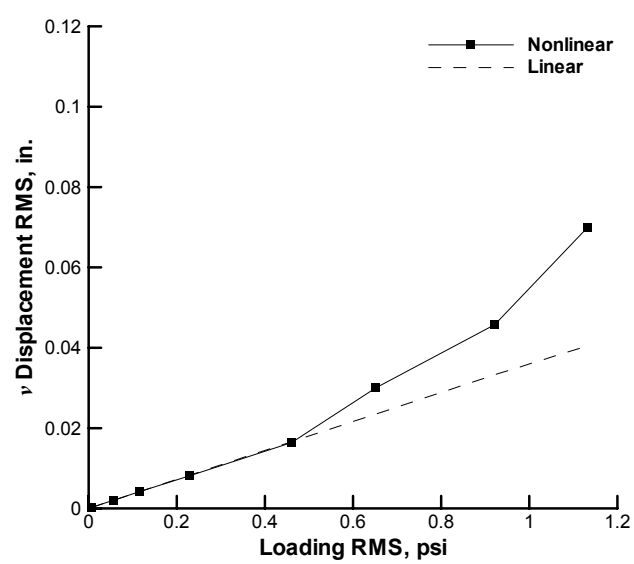

Figure 11: Quarter-span $v$ displacement RMS. 


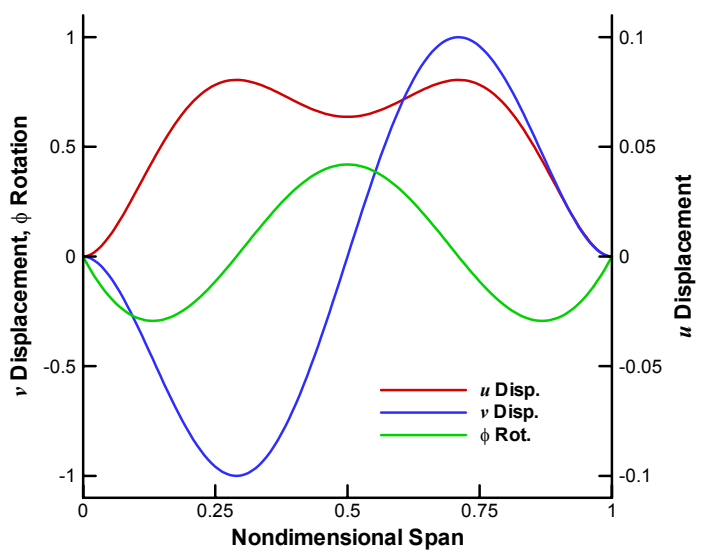

Figure 12: The first type $\mathrm{T}$ anti-symmetric transverse mode AT- $\mathrm{T}_{1}(158 \mathrm{~Hz})$.

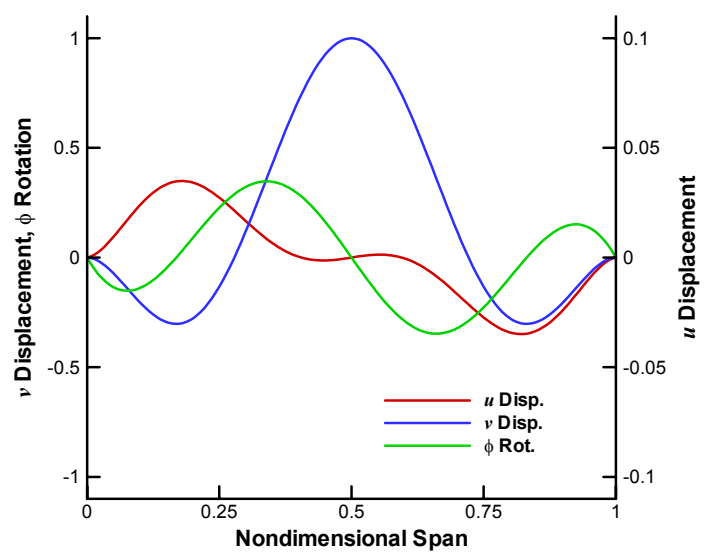

Figure 13: The first type $\mathrm{T}$ symmetric transverse mode ST- $\mathrm{T}_{1}(258 \mathrm{~Hz})$.

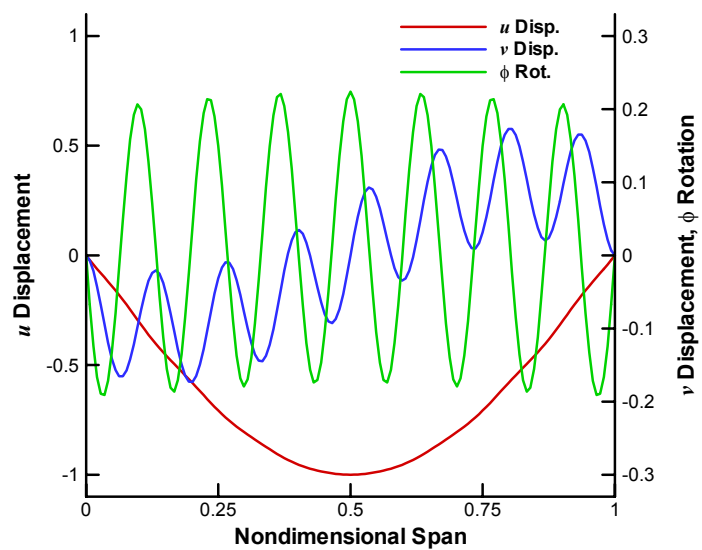

Figure 14: The first type I anti-symmetric transverse mode AT- $I_{1}(5.6 \mathrm{kHz})$.

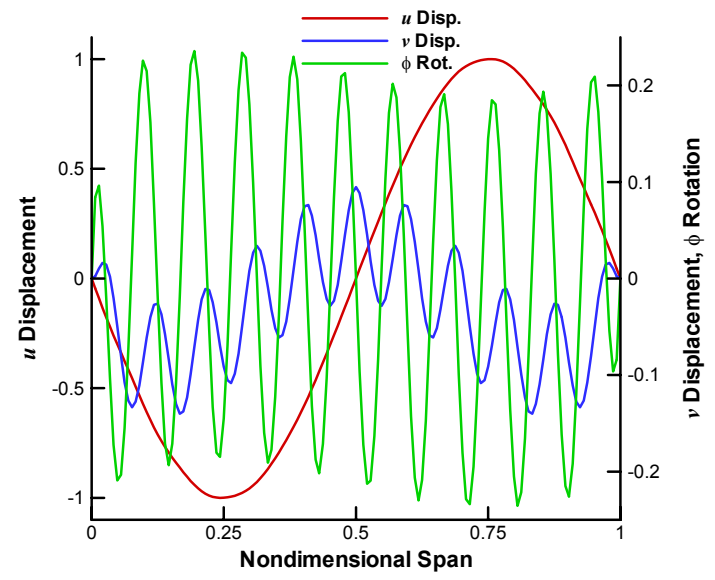

Figure 15: The first type I symmetric transverse mode $\mathrm{ST}^{-\mathrm{I}_{1}}(11.2 \mathrm{kHz})$.

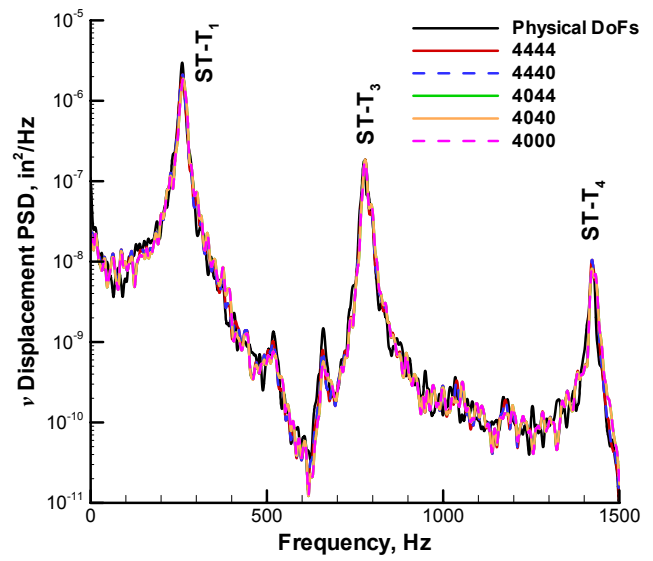

Figure 16: Mid-span vertical displacement at $0.2304 \mathrm{lb} / \mathrm{in}$. distributed loading. ${ }^{*}$

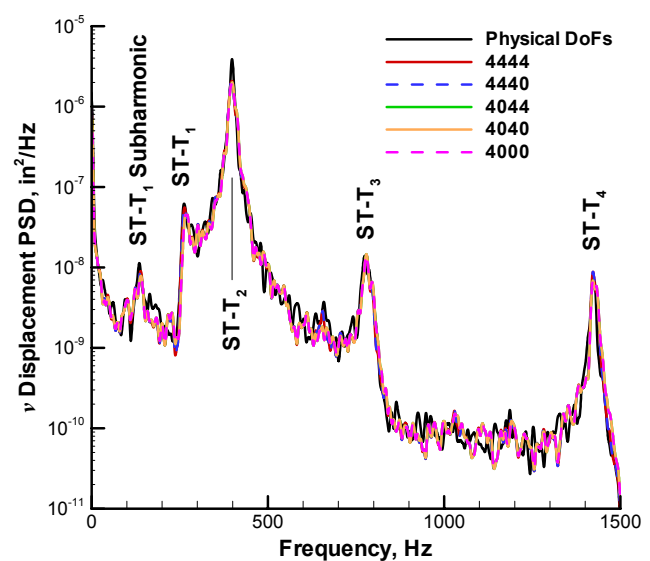

Figure 17: Quarter-span vertical displacement at $0.2304 \mathrm{lb} /$ in. distributed loading.

* Modal basis order: ST-T, ST-I, AT-T, AT-I. 


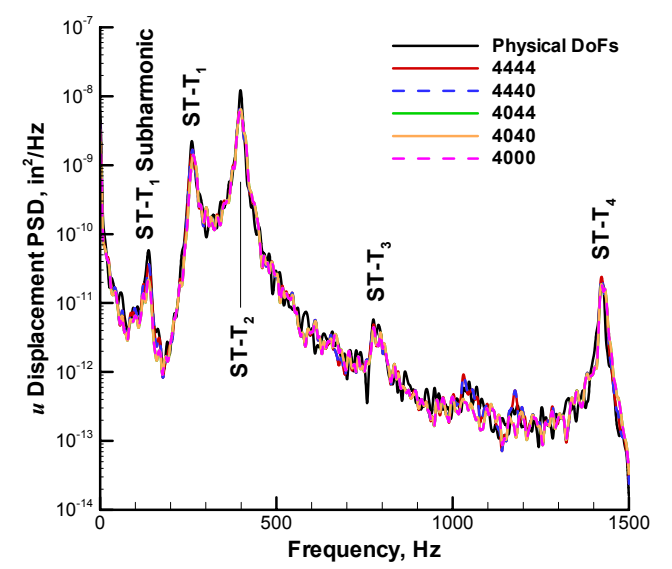

Figure 18: Quarter-span horizontal displacement at $0.2304 \mathrm{lb} /$ in. distributed loading. ${ }^{*}$

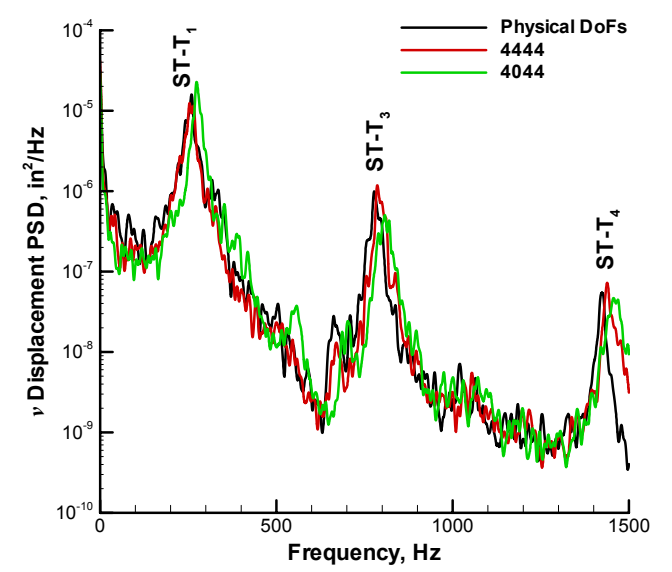

Figure 19: Mid-span vertical displacement at $0.6517 \mathrm{lb} / \mathrm{in}$. distributed loading. ${ }^{*}$

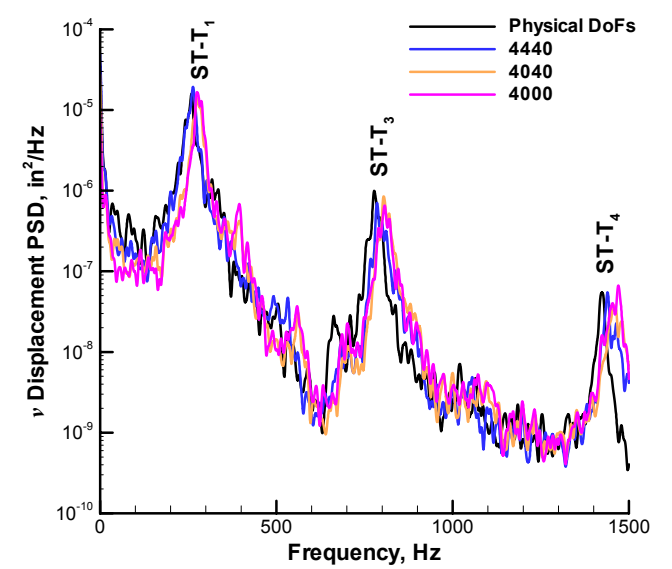

Figure 20: Mid-span vertical displacement at $0.6517 \mathrm{lb} /$ in. distributed loading. ${ }^{*}$

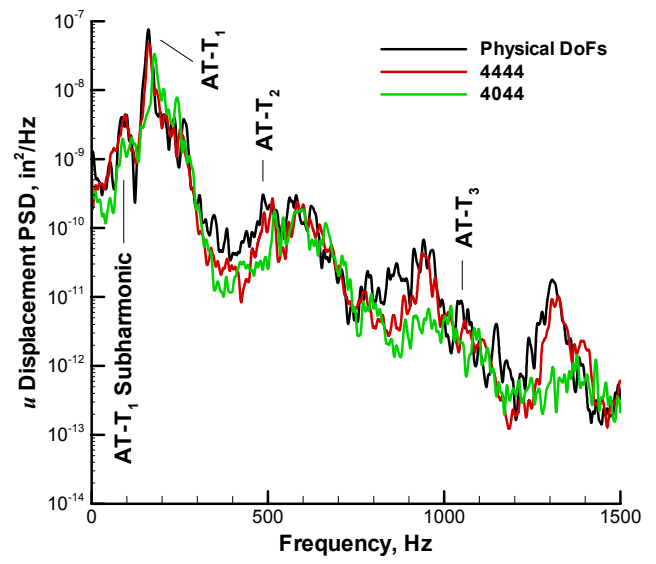

Figure 21: Mid-span horizontal displacement at $0.6517 \mathrm{lb} /$ in. distributed loading.

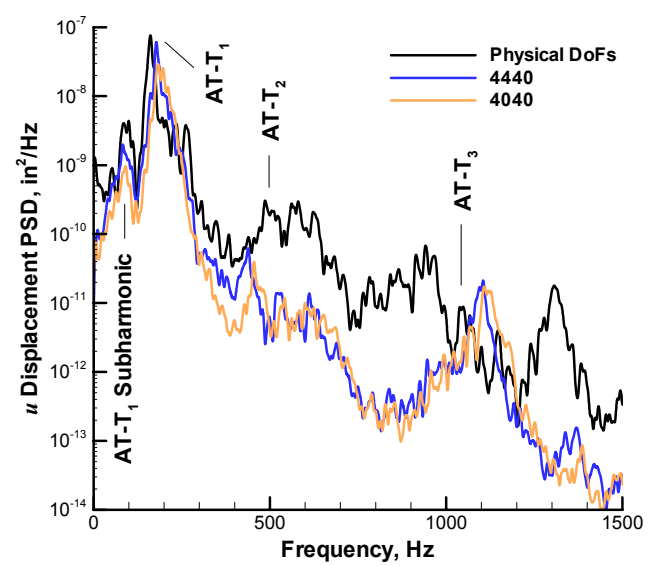

Figure 22: Mid-span horizontal displacement at $0.6517 \mathrm{lb} /$ in. distributed loading.

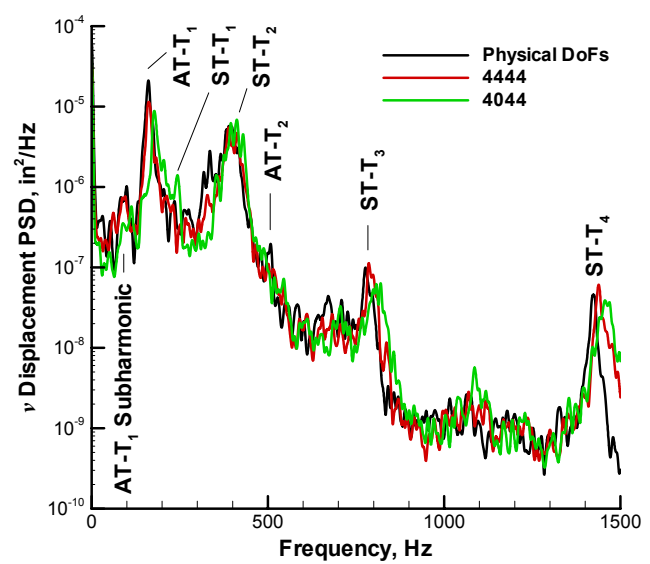

Figure 23: Quarter-span vertical displacement at $0.6517 \mathrm{lb} /$ in. distributed loading.

* Modal basis order: ST-T, ST-I, AT-T, AT-I. 


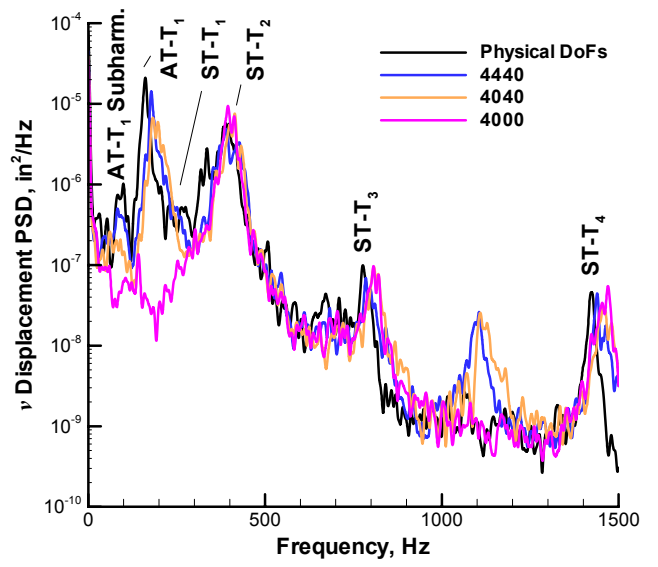

Figure 24: Quarter-span vertical displacement at $0.6517 \mathrm{lb} / \mathrm{in}$. distributed loading.

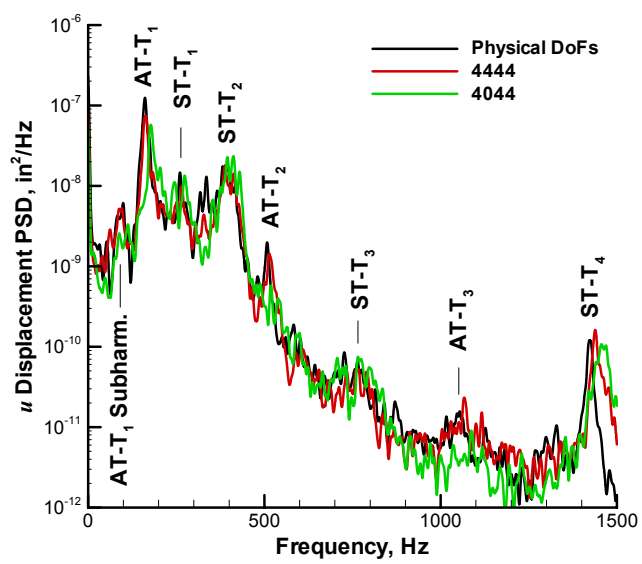

Figure 25: Quarter-span horizontal displacement at $0.6517 \mathrm{lb} /$ in. distributed loading.

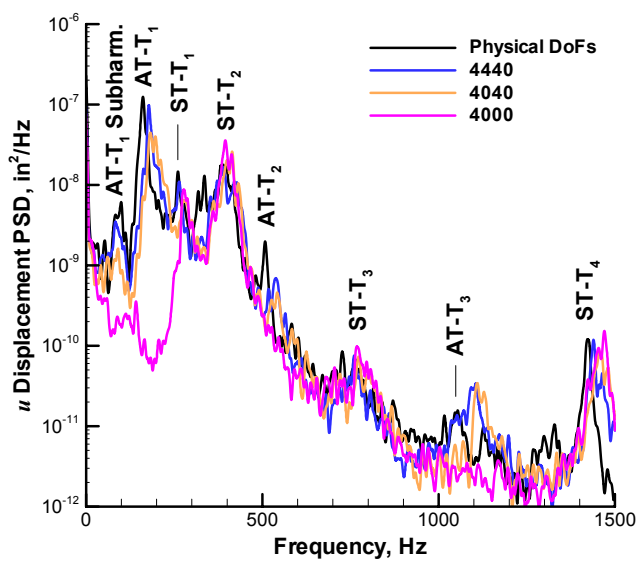

Figure 26: Quarter-span horizontal displacement at $0.6517 \mathrm{lb} /$ in. distributed loading.

* Modal basis order: ST-T, ST-I, AT-T, AT-I.
Table 1: Natural modes - classification and frequencies.

\begin{tabular}{|c|c|c|}
\hline $\begin{array}{c}\text { Mode } \\
\text { Designator }\end{array}$ & $\begin{array}{c}\text { Mode } \\
\text { Number }\end{array}$ & $\begin{array}{c}\text { Frequency } \\
\quad(\mathbf{H z})\end{array}$ \\
\hline $\mathrm{ST} \mathrm{T}_{1}$ & 2 & 258.17 \\
\hline $\mathrm{ST}-\mathrm{T}_{2}$ & 3 & 400.41 \\
\hline $\mathrm{ST}_{-} \mathrm{T}_{3}$ & 5 & 773.72 \\
\hline $\mathrm{ST}_{-} \mathrm{T}_{4}$ & 7 & $1,426.0$ \\
\hline ST-I ${ }_{1}$ & 22 & 11,225 \\
\hline ST-I $I_{2}$ & 33 & 22,437 \\
\hline $\mathrm{ST}^{-\mathrm{I}_{3}}$ & 42 & 33,638 \\
\hline ST-I $\mathrm{I}_{4}$ & 51 & 44,826 \\
\hline $\mathrm{AT} \mathrm{T}_{1}$ & 1 & 158.25 \\
\hline $\mathrm{AT}-\mathrm{T}_{2}$ & 4 & 513.19 \\
\hline $\mathrm{AT}-\mathrm{T}_{3}$ & 6 & 1070.1 \\
\hline $\mathrm{AT}-\mathrm{T}_{4}$ & 8 & 1828.0 \\
\hline AT-I ${ }_{1}$ & 15 & $5,624.3$ \\
\hline AT- $\mathrm{I}_{2}$ & 28 & 16,831 \\
\hline $\mathrm{AT}-\mathrm{I}_{3}$ & 38 & 28,039 \\
\hline $\mathrm{AT}-\mathrm{I}_{4}$ & 47 & 39,235 \\
\hline
\end{tabular}

Table 2: Selected nonlinear cross stiffness coefficients.

\begin{tabular}{cccc}
\hline \hline Modes & $a_{j k}$ & $b_{j j k}$ & $b_{j k k}$ \\
\hline $\begin{array}{c}\text { ST-T } \\
\text { ST-I }\end{array}$ & $6.80 \mathrm{E}+10$ & $3.73 \mathrm{E}+11$ & $-8.46 \mathrm{E}+11$ \\
\hline $\begin{array}{c}\text { AT-T } \\
\text { AT-I }\end{array}$ & $3.20 \mathrm{E}-01$ & $1.43 \mathrm{E}+11$ & $-1.86 \mathrm{E}+12$ \\
\hline $\begin{array}{c}\text { ST-T } \\
\text { AT-T }_{1}\end{array}$ & $-1.46 \mathrm{E}+04$ & $-2.09 \mathrm{E}+08$ & $2.74 \mathrm{E}+12$ \\
\hline $\begin{array}{l}\text { ST-I } \\
\text { AT-I }_{1}\end{array}$ & $1.05 \mathrm{E}+10$ & $-4.60 \mathrm{E}+11$ & $-4.44 \mathrm{E}+04$ \\
\hline $\begin{array}{l}\text { ST-T } \\
\text { AT-I }\end{array}$ & $2.24 \mathrm{E}+04$ & $-3.21 \mathrm{E}+08$ & $6.66 \mathrm{E}+11$ \\
\hline $\begin{array}{r}\text { AT-T } \\
\text { ST-I }\end{array}$ & $3.70 \mathrm{E}+10$ & $1.00 \mathrm{E}+03$ & $-2.90 \mathrm{E}+09$ \\
\hline \hline
\end{tabular}

